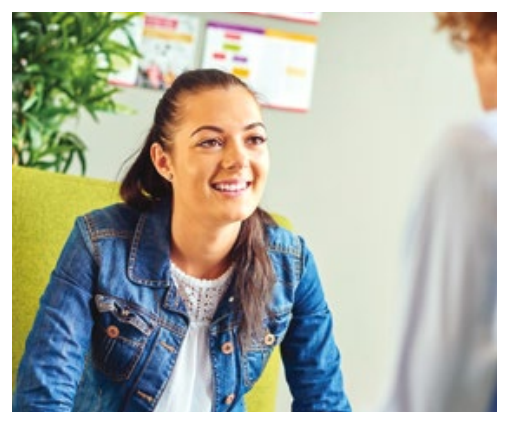

\title{
Human papillomavirus testing as part of the renewed National Cervical Screening Program
}

CPD

\section{David Hawkes}

\section{Background}

On 1 December 2017, Australia moved to a new National Cervical Screening Program (NCSP), which uses primary human papillomavirus (HPV) nucleic acid testing (NAT) followed by reflex liquid-based cytology for women aged between 25 and 74 years.

\section{Objectives}

The aim of this article is to provide an overview of the different HPV NAT assays that satisfy the requirements for use in the renewed NCSP.

\section{Discussion}

Australia has adopted innovative, evidence-based criteria for the inclusion of HPV NAT assays in the renewed NCSP. These include the requirements for detection of all 12 designated oncogenic HPV types, including separate detection and reporting of HPV 16 and 18; validation against reference assays showing sufficient sensitivity and specificity for the detection of underlying highgrade cervical disease; reproducibility; and the presence of cellularity and inhibition controls. Practitioners can feel assured that HPV NAT undertaken as part of the renewed NCSP will produce high-quality results irrespective of location or pathology provider.
THE Renewed Australian National Cervical Screening Program (NCSP) began on 1 December 2017. Recommendations made in 2014 by the Medical Services Advisory Committee (MSAC), which examined new evidence including technologies, form the basis of the renewed NCSP. While the Pap smear at two-yearly intervals has been the basis of the Australian NCSP for over 25 years, the key recommendation of the MSAC was that the Pap smear be replaced by a cervical screening test (CST) that would use a human papillomavirus (HPV) test as the primary screening test followed by reflex cytology of HPV-positive specimens. ${ }^{1}$ The renewed NCSP also includes an increase in the screening entry age from 18 to 25 years and screens women until the age of 69 years, with an exit test between 70 and 74 years. The renewed NCSP has also introduced the option for women who are under-screened or who have never participated in cervical screening to access HPV self-collection in a clinical setting.

\section{HPV NAT}

There is a wealth of evidence showing the increased sensitivity of HPV-based screening, compared with cytology-based screening. ${ }^{2-4}$ A population-based HPV primary cervical screening program began in the Netherlands earlier in 2017. In the Netherlands there was a tender process to determine a single HPV nucleic acid testing (NAT) assay that would be used throughout the country. Australia has taken a different approach by using a quality-based requirements framework for testing, which allows different pathology providers to select the assay that best fits their needs as long as it meets the performance standards and characteristics required by the program.

\section{Requirements for the use of human papillomavirus nucleic HPV NAT assays in the National Cervical Screening Program}

In Australia, the type of HPV NAT that can be undertaken in the NCSP is governed by the National Pathology Accreditation Advisory Council (NPAAC) requirements, specifically those for laboratories reporting tests for the NCSP. ${ }^{5}$ These requirements have been designed to reduce the risk of false results occurring, either positive or negative.

\section{Oncogenic HPV}

There are $12 \mathrm{HPV}$ types classified as definitely oncogenic $(16,18,31,33,35$, $39,45,51,52,56,58,59)$ according to the World Health Organization. ${ }^{6}$ NPAAC requires that all assays used in the NCSP are commercially supplied assays that can detect all designated 12 oncogenic HPV types. HPV assays must also be able to separately identify HPV 16 and HPV 18 in order to identify women who are at higher risk of developing cervical cancer on the basis solely of the HPV result. Some assays give a combined result for HPV 18 and HPV $45(18 / 45)$, and these assays are regarded as satisfying the requirements, with women who test positive for HPV 18/45 undertaking the same clinical pathway as women who test positive for HPV 18. 


\section{Clinical sensitivity, specificity and reproducibility}

HPV NAT assays have a clinical cut-off point for positivity, which has been set specifically to facilitate their use as a riskstratification test for primary screening. HPV NAT assays are not a diagnostic test for cervical disease, nor are they a diagnostic test for the presence of any HPV at all; that is, the cut-off point requires a threshold amount of HPV to be present. This threshold amount is associated with the presence of a high-grade squamous intraepithelial lesion (HSIL). Many HPV infections either resolve without treatment or may persist but never lead to cervical abnormalities. Additionally, there is currently no treatment for HPV infections per se, other than the removal of HPVpositive lesions. The clinical sensitivity, specificity and reproducibility of HPV NAT assays are assessed using a protocol known as the Meijer criteria. ${ }^{7}$ Briefly, an HPV NAT assay must show clinical sensitivity and specificity for detecting the underlying presence of a cervical HSIL of not less than $90 \%$ and $98 \%$ respectively of a validated reference assay. To satisfy the Meijer criteria, an HPV NAT assay must also show intra-laboratory and inter-laboratory agreement with a lower confidence bound of $87 \%$.

\section{HPV NAT assay controls}

In addition to showing clinical sensitivity, specificity and reproducibility, the NPAAC requirements state that an HPV NAT assay must contain a control to monitor for inhibition and/or assay failure and a control for cellularity to detect inadequate or empty cervical samples. The control for inhibition/assay failure is critically important, as contaminants of cervical samples, such as blood or lubricant, can inhibit the ability of an assay to detect HPV. The cellularity control is also important, as an assay lacking a cellularity control would report an empty liquidbased cytology vial with insufficient or absent cellular material as negative, and a recommendation to rescreen in five years may then be incorrectly assigned to a woman. Unsatisfactory samples - those with low cellularity (or inhibition) - occur at a rate of $0.1-0.2 \%$ in clinician-collected samples, but this rate has been observed to be as high as $10 \%$ in some self-collection studies from overseas. Within the Australian environment, a recent pilot study of the renewed NCSP guidelines for self-collection in Victoria found the rate of unsatisfactory self-collected samples, resulting from low cellularity, was $2.5 \%{ }^{8}$ A recent examination of unsatisfactory results in cytology-based CSTs in Victoria found a rate of $2.7 \% .^{9}$

\section{HPV NAT for self-collected samples}

As part of the renewed NCSP, an alternative screening pathway will be offered to eligible under-screened or never-screened women attending a healthcare setting to overcome the barriers some women experience with having a clinician-collected CST. Eligible women must:

- be over 30 years of age and at least two or more years overdue for a CST

- have declined a speculum examination

- be under the supervision of a health professional that routinely offers cervical screening.

There is a wealth of data showing that a self-collected sample tested for HPV NAT ${ }^{10}$ is of similar sensitivity for detecting HSIL as a clinician-collected sample. However, it has also been noted that PCR-based tests have stronger evidence of equivalence to cliniciancollected samples and, as such, the NPAAC requirements clearly state that any self-collected specimens must be tested on a PCR-based HPV test. Currently, only one pathology laboratory in Australia is validated for testing of self-collected samples for HPV NAT. ${ }^{11}$

\section{Other quality measures}

In addition to the requirements that an HPV NAT assay must meet for use within the NCSP described above, there are a number of additional quality control measures in place. Each laboratory has to undertake a minimum number of tests in order to accurately monitor the variation in HPV-positive results being produced at other laboratories and ensure sufficient consistency with national rates. It is hoped that this will ensure that any potential quality issues that could adversely affect assay performance - for example, variation in liquid-based cytology media or storage/transport conditions - will be detected prior to disposal of patient specimens.

The HPV NAT assays are also subject to a range of quality-control processes. For example, the NPAAC requires that controls not supplied by the manufacturer are run on every day that HPV is being tested for. Additionally, laboratories performing HPV NAT assays must participate in an external quality assurance program (eg the Royal Australasian College of Pathologists Quality Assurance Program), and any discrepancies must be investigated.

\section{HPV NAT assays used in the renewed National Cervical Screening Program}

At the time of writing, there were six HPV NAT assays that had met the requirements for use in the renewed NCSP (Table 1). The Roche cobas 4800 and Roche cobas 6800 HPV NAT assays are the most widely used throughout Australia. All six are in use in Australian laboratories in some form. The Australian requirements are based on an assay satisfying certain quality measures and, as such, a number of other assays are likely to be available for use in the near future, including assays from Hologic, AusDiagnostics and Euroimmun. This flexibility will allow pathology laboratories to choose HPV NAT assays that best meet the needs of their referring practitioners and workflows.

\section{Conclusion}

Australia has adopted innovative, evidence-based criteria for the inclusion of HPV NAT assays in the renewed NCSP. In addition, the quality of HPV testing in the program is further supported by a comprehensive quality program, which includes monitoring of HPV positivity rates in CSTs and the daily testing of quality control samples. Practitioners 
Table 1. NPAAC requirements for HPV assays for use in the renewed National Cervical Screening Program

\begin{tabular}{|c|c|c|c|c|c|}
\hline \multirow{2}{*}{ HPV assay } & \multicolumn{5}{|c|}{ NPAAC requirements } \\
\hline & Meijer criteria & Screening & Cellularity & Inhibition & $\frac{\text { Self-collection }}{\text { PCR-based }}$ \\
\hline Roche cobas 4800 & $\checkmark$ & $\checkmark$ & $\checkmark$ & $\checkmark$ & $\checkmark$ \\
\hline Abbott RealTime High-Risk HPV & $\checkmark$ & $\checkmark$ & $\checkmark$ & $\checkmark$ & $\checkmark$ \\
\hline Seegene Anyplex II & $\checkmark$ & $\checkmark$ & $\checkmark$ & $\checkmark$ & $\checkmark$ \\
\hline Cepheid GeneXpert & $\checkmark$ & $\checkmark$ & $\checkmark$ & $\checkmark$ & $\checkmark$ \\
\hline Roche cobas 6800 & $\checkmark$ & $\checkmark$ & $\checkmark$ & $\checkmark$ & $\checkmark$ \\
\hline
\end{tabular}

HPV, human papillomavirus, NPAAC, National Pathology Accreditation Advisory Council

can feel assured that HPV NAT undertaken as part of the renewed NCSP will produce high-quality results irrespective of location or pathology provider.

\section{Author}

David Hawkes BSc (Hons), PhD, Director, Molecular Biology and Biochemistry Victorian Cytology Service (VCS), Melbourne, Vic; Honorary Research Fellow, Department of Pharmacology and Therapeutics, University of Melbourne Vic. dhawkes@vcs.org.au Competing interests: $\mathrm{DH}$ is an investigator on the Compass Trial for which VCS has received funding from Roche Molecular Diagnostics, but has never received any personal financial benefits.

Provenance and peer review: Not commissioned, externally peer reviewed.

\section{References}

1. Medical Services Advisory Committee. MSAC outcomes. Application No. 1276 - Renewal of the National Cervical Screening Program. Canberra: Commonwealth of Australia, 2014.

2. Dillner J, Rebolj M, Birembaut $\mathrm{P}$, et al. Long term predictive values of cytology and human papillomavirus testing in cervical cancer screening: Joint European cohort study. BMJ 2008:337:a1754. doi: 10.1136/bmj.a1754.

3. Khan MJ, Castle PE, Lorincz AT, et al. The elevated 10-year risk of cervical precancer and cancer in women with human papillomavirus (HPV) type 16 or 18 and the possible utility of type-specific HPV testing in clinical practice. J Natl Cancer Inst 2005;97(14):1072-79.

4. Canfell K, Caruana M, Gebski V, et al. Cervical screening with primary HPV testing or cytology in a population of women in which those aged 33 years or younger had previously been offered HPV vaccination: Results of the Compass pilot randomised trial. PLoS Med 2017;14(9):e1002388. doi: 10.1371/journal.pmed.1002388.

5. National Pathology Accreditation Advisory Council (NPAAC). The requirements for laboratories reporting tests for the National Cervical Screening Program. Canberra: NPAAC, 2017.
6. Bouvard V, Baan R, Straif $K$, et al. A review of human carcinogens - Part B: Biological agents. Lancet Oncol 2009;10(4):321-22.

7. Meijer CJ, Berkhof J, Castle PE, et al. Guidelines for human papillomavirus DNA test requirements for primary cervical cancer screening in women 30 years and older. Int J Cancer 2009;124(3):516-20. doi: 10.1002/ijc.24010.

8. Saville M, Hawkes D, McLachlan E, Anderson S, Arabena K. Self-collection for under-screened women in a National Cervical Screening Program: Pilot study. Curr Oncol 2018:25(1):e27-e32. doi: 10.3747/co.25.3915

9. Victorian Cervical Cytology Registry. Statistical report 2014. Carlton South, Vic: VCCR, 2014.

10. Arbyn M, Verdoodt F, Snijders PJ, et al. Accuracy of human papillomavirus testing on selfcollected versus clinician-collected samples: A meta-analysis. Lancet Oncol 2014;15(2):172-83. doi: 10.1016/S1470-2045(13)70570-9.

11. Hayes P. Cervical self-sampling for under-screened women now available. East Melbourne: NewsGP RACGP, 2018. Available at www.racgp.org.au/ newsGP/Clinical/Cervical-self-sampling-for-underscreened-women-no [Accessed 23 April 2018]. 